\title{
Risco de doenças cardiovasculares com perfil intestinal influenciado pela deficiência de vitamina $D$
}

\author{
Risk of cardiovascular diseases with intestinal profile influenced by vitamin D deficiency \\ Riesgo de enfermedades cardiovasculares con perfil intestinal influenciado por deficiencia de \\ vitamina D
}

Recebido: 24/05/2021 | Revisado: 29/05/2021 | Aceito: 29/05/2021 | Publicado: 02/06/2021

\author{
Andressa Araújo de Souza \\ ORCID: https://orcid.org/0000-0003-4469-9565 \\ Centro Universitário Fametro, Brasil \\ E-mail: andressaaraujo538@gmail.com \\ Marcileide da Silva Veras \\ ORCID: https://orcid.org/0000-0002-2602-4073 \\ Centro Universitário Fametro, Brasil \\ E-mail: eumarcy.silva@gmail.com \\ Mayra Tereza Aguiar Pantoja \\ ORCID: https://orcid.org/0000-0001-8123-1684 \\ Centro Universitário Fametro, Brasil \\ E-mail: mayraaguiar98@gmail.com \\ José Carlos de Sales Ferreira \\ ORCID: https://orcid.org/0000-0002-1867-8229 \\ Centro Universitário Fametro, Brasil \\ E-mail: jcarlos.sales@gmail.com
}

\begin{abstract}
Resumo
Introdução: as DCNT's retratam um grande e importante problema de saúde pública em todos os países, dentre essas doenças estão as cardiovasculares (DCV), responsáveis por uma grande taxa de mortalidade mundialmente. Objetivo: Deste modo, o presente estudo tem como finalidade descrever achados da literatura que estabeleçam a relação entre a deficiência de vitamina $\mathrm{D}$, com o desiquilíbrio da microbiota e o desenvolvimento de doenças cardiovasculares. Metodologia: A metodologia utilizada foi uma revisão bibliográfica de caráter narrativa. A coleta de dados foi realizada em sites como Scielo (Scientific Electronic Library Online), PubMed (Banco de Dados de Publicações Médicas) e Google Acadêmico nos idiomas português e inglês. Foram incluídos artigos, teses, monografias e sites que apresentavam conteúdos e títulos que se enquadraram no tema estudado e no período de tempo de 2011 a 2021 , na língua portuguesa ou de demais línguas como a inglesa, e excluídos aqueles que não se enquadravam nesses critérios. Resultados e discursões: Existem indícios da participação da vitamina D na homeostase do sistema cardiovascular por mediação da regulação microbiota e do estado inflamatório. Conclusão: Mesmo com os estudos atuais ainda são necessários mais estudos e pesquisas que avaliem tal relação de maneira que esclareça os processos envolvendo a vitamina D, microbiota e sistema cardiovascular e seus respectivos papéis no risco cardiovascular aumentado, para que se possa estabelecer não apenas futuras linhas de tratamento, mas também meios de prevenção.
\end{abstract}

Palavras-chave: Vitamina D; Doenças cardiovasculares; Intestino.

\begin{abstract}
Introduction: as NCDs portray a major and important public health problem in all countries, among these diseases are cardiovascular diseases (CVD), responsible for a large mortality rate worldwide. Objective: Thus, the present study aims to describe findings in the literature that establish the relationship between vitamin D deficiency, with the imbalance of the microbiota and the development of cardiovascular diseases. Methodology: The methodology used was a literature review of narrative character. Data collection was carried out on sites such as Scielo (Scientific Electronic Library Online), PubMed (Database of Medical Publications) and Google Scholar in Portuguese and English. Articles, theses, monographs and websites were included that presented contents and titles that fit the studied theme and in the period from 2011 to 2021, in Portuguese or other languages such as English, and those that did not meet these criteria were excluded. . Results and speeches: There are indications of the participation of vitamin D in homeostasis of the cardiovascular system through the mediation of microbiota regulation and the inflammatory state. Conclusion: Even with current studies, more studies and research are needed to evaluate such a relationship in order to clarify the processes involving vitamin D, microbiota and cardiovascular system and their respective roles in increased cardiovascular risk, so that it is possible to establish not only future one's lines of treatment, but also means of prevention.
\end{abstract}

Keywords: Vitamin D; Cardiovascular diseases; Intestine. 


\begin{abstract}
Resumen
Introducción: las ECNT representan un importante e importante problema de salud pública en todos los países, entre estas enfermedades se encuentran las enfermedades cardiovasculares (ECV), responsables de una alta tasa de mortalidad a nivel mundial. Objetivo: Así, el presente estudio tiene como objetivo describir hallazgos en la literatura que establecen la relación entre la deficiencia de vitamina $\mathrm{D}$, con el desequilibrio de la microbiota y el desarrollo de enfermedades cardiovasculares. Metodología: La metodología utilizada fue una revisión bibliográfica de carácter narrativo. La recolección de datos se llevó a cabo en sitios como Scielo (Scientific Electronic Library Online), PubMed (Base de datos de publicaciones médicas) y Google Scholar en portugués e inglés. Se incluyeron artículos, tesis, monografías y sitios web que presentaban contenidos y títulos que se ajustan a la temática estudiada y en el período de 2011 a 2021, en portugués u otros idiomas como el inglés, y se excluyeron aquellos que no cumplían con estos criterios. Resultados y discursos: Existen indicios de la participación de la vitamina D en la homeostasis del sistema cardiovascular a través de la mediación de la regulación de la microbiota y el estado inflamatorio. Conclusión: Incluso con los estudios actuales, se necesitan más estudios e investigaciones para evaluar esta relación con el fin de aclarar los procesos que involucran la vitamina $\mathrm{D}$, la microbiota y el sistema cardiovascular y sus respectivos roles en el aumento del riesgo cardiovascular, de modo que no solo se puedan establecer futuros tratamientos. líneas, sino también medios de prevención.
\end{abstract}

Palabras clave: Vitamina D; Enfermedades cardiovasculares; Intestino.

\title{
1. Introdução
}

As doenças crônicas não transmissíveis (DCNT's) retratam um grande e importante problema de saúde pública nos países desenvolvidos e em desenvolvimento, dentre essas doenças estão as cardiovasculares (DCV), que são responsáveis por uma grande taxa de mortalidade em todo o mundo (Sousa, 2018). Estas afetam o sistema circulatório, isto é, o coração e os vasos sanguíneos dentre eles veias, vasos, as artérias e capilares, acarretando ocorrências graves que ocasionam de uma trombose venosa profunda (TVP), infarto agudo do miocárdio (IAM) ao acidente vascular cerebral (AVC) entre outras (Bourbon, 2016).

De acordo com avaliações da Organização Mundial da Saúde (OMS) indicam que as DCNT são culpadas por $71 \%$ das 57 milhões de mortes ocorridas mundialmente em 2016 (Brasil, 2020). No Brasil, as DCNT são igualmente relevantes, tendo sido responsáveis, em 2016, por 74\% do total de mortes, com destaque para doenças cardiovasculares (28\%), neoplasias (18\%), doenças respiratórias (6\%) e diabetes (5\%) (Brasil, 2020). No mundo 17 milhões de pessoas são acometidos por problemas no coração. E no Brasil, esse número anual chega atingir 300 mil, conforme o Ministério da Saúde, o que equivale a um óbito a cada dois minutos. O quantitativo de 3,5 milhões de mortes em uma década, datadas de 2004 a 2014 , foram provocadas por complicações no coração e na circulação sanguínea. Número que indica quase mil mortes diárias (Brasil, 2017).

Atualmente diversas publicações têm chamado à atenção sobre a importância do status de vitamina $\mathrm{D}$ e da diversidade da microbiota intestinal desde o início da vida para a ocorrência de doenças (Ly et al, 2011; Weiss, 2011; Weiss \& Litonjua, 2015). E de acordo com Luthold (2017), investigações sobre o envolvimento da microbiota intestinal entre os mecanismos pelos quais a deficiência de vitamina D se associa a doenças cardiometabólicas se justificaram pelo fato destas apresentarem inflamação subclínica como base fisiopatológica.

Outros estudos com pacientes autoimunes também apresentaram uma interação direta da vitamina D com a microbiota intestinal, ocasionando a melhora no quadro de disbiose nesses pacientes. Sendo os indivíduos com apropriado nível sérico de 25(OH)D foram associados com menor risco de infecção por Clostridium, quando comparados com indivíduos com deficiência da vitamina (Ananthakrishnan et al., 2014).

E ainda, a vitamina D além de agir de maneira indireta sobre o perfil de risco cardiovascular pelas ações no metabolismo, as próprias células do sistema circulatório (miócitos cardíacos e as células produtoras de renina, endoteliais e musculares lisas dos vasos sanguíneos) expressam o citocromo P450 família 27 subfamílias membro B 1 (CYP27B1) e/ou o receptor da vitamina $\mathrm{D}$ (VDR). A $1,25(\mathrm{OH})_{2} \mathrm{D}$ participa do controle da função cardíaca e da pressão arterial por meio da 
regulação do crescimento das células musculares e da contratilidade miocárdica (Castro, 2011; Norman \& Powell., 2014). E há pouco tempo, constatou-se que níveis de vitamina $\mathrm{D}$ inadequados na infância também podem exercer influência no risco de aterosclerose na vida adulta (Juonala et al., 2015).

Embora existam inúmeras evidências experimentais e clínicas acerca da relação entre a vitamina $\mathrm{D}$, e em particular da sua carência, e o acrescimento do risco cardiovascular, não é possível concluir se esta associação apresenta uma relação causal (Nadir, Szwejkowski \& Witham, 2010; Santos., 2011). Assim, esta questão necessita de estudos mais profundos, sendo que apenas após a análise dos mais resultados se poderá efetuar alguma conclusão sobre o benefício da suplementação com vitamina D como tratamento base na prevenção primária e secundária de doenças cardiovasculares (Santos, 2011). Deste modo, o presente estudo tem como finalidade descrever achados da literatura que estabeleçam a relação entre a deficiência de vitamina $\mathrm{D}$, com o desiquilíbrio da microbiota e o desenvolvimento de doenças cardiovasculares.

\section{Metodologia}

O estudo desenvolvido trata-se de uma revisão bibliográfica de caráter narrativa. De acordo com Rother (2007) a revisão narrativa vale-se de fontes de informações bibliográficas ou eletrônicas para o alcance de resultados de pesquisas de outros autores, com a finalidade de fundamentar teoricamente um determinado objetivo.

A coleta de dados em busca de literatura foi realizada em livros, revistas, sites oficiais e artigos, teses e monografias estes pesquisados em sites como Scielo (Scientific Electronic Library Online), PubMed (Banco de Dados de Publicações Médicas) e Google Acadêmico nos idiomas português e inglês. Para as buscas nas plataformas foram utilizados os seguintes descritores em português e inglês respectivamente: intestino, vitamina $\mathrm{D}$, doenças cardiovasculares, relação da vitamina $\mathrm{D}$ e doenças cardiovasculares, perfil intestinal, influenciado pela falta de vitamina $\mathrm{D}$, fatores de risco da hipovitaminose $\mathrm{D}$, doenças cardiovasculares e vitamina $\mathrm{D}$, doenças cardiovasculares e a relação com o intestino e vitamina $\mathrm{D}$; intestine, vitamin $D$, cardiovascular diseases, related to vitamin $D$ and cardiovascular diseases, intestinal profile influenced by the lack of vitamin $D$, risk factors of hypovitaminosis $D$, cardiovarcular diseases and vitamin $D$, cardiovascular diseases related with the intestine and vitamin $D$.

Para análise de dados foi definido o período dos últimos dez anos, de 2011 a 2021. Como critérios de exclusão foram utilizados os seguintes: títulos que fugiam ao tema abordado; artigos, teses e monografias que não estavam dentro do período estabelecido assim como conteúdos de livros, revistas e sites. E como critérios de inclusão foram utilizados: conteúdos e títulos que se enquadraram no tema estudado e no período estabelecido sendo eles da língua portuguesa ou de demais línguas como a inglesa.

\section{Resultados e Discussão}

\subsection{Funções da vitamina D no organismo}

Vitaminas são substâncias orgânicas essenciais que não podem ser sintetizados pelo corpo humano e devem ser ingeridos. O termo vitamina D relaciona-se à vitamina D2 (ergosterol) que é obtida de fontes vegetais da alimentação e suplementos orais e vitamina D3 (colecalciferol) que é principalmente a partir de exposição da pele à radiação ultravioleta B (RUVB) presente na luz solar e pela ingestão de alimentos, tais como fontes de óleo de peixe e alimentos fortificados mortificável (leite, sumos, margarinas, iogurtes, cereais e soja) e suplementos orais, uma vez que são as versões mais abundantes e ativas (Kennel, 2010). 
Poucos alimentos contêm naturalmente ou são enriquecidos com vitamina D. Consequentemente, sem consumo regular diário de alimentos naturalmente ricos ou enriquecidos, os indivíduos podem desenvolver deficiência de vitamina D. $\mathrm{Na}$ ausência de exposição diária à luz solar, ou com o uso de filtros solares, esta deficiência será ainda maior (Filho \& Melamed, 2013).

De acordo Zittermann e Gummert (2010) as ações celulares da vitamina D são intercedidas por um receptor de membrana e nuclear da vitamina D (VDR). Este receptor se liga a sua forma ativa que é o 1,25 dihidroxicolecalciferol (25OH2-D) (Rhodes et al., 2020). Uma proteína com homologia estrutural e funcional a um largo espectro de receptores nucleares hormonais, designadamente das hormonas tiroideia, ácido retinóico, esteroides, bem como de receptores endógenos de fatores de transcrição (Shoback, Sellmeyer \& Bikleeste, 2011). Este receptor está presente na maior parte das células e tecidos do organismo, pelo que é possível achar uma resposta generalizada a exposição à vitamina D. Aproximadamente 3\% do genoma humano é regulado, direta ou indiretamente, pelo sistema endócrino da vitamina D (Zittermann \& Gummert, 2010).

Foi estabelecido, nas últimas quatro décadas, que o sistema endócrino da vitamina $\mathrm{D}$ é deliberado pela presença do VDR, é operacional em, pelo menos, 38 tecidos do corpo humano que o expressam, especificamente no tecido adrenal, ósseo (osteoblastos), adiposo, cerebral (hipotálamo, células da glia, amígdala), na mama, cartilagem, epidídimo (túbulos seminíferos), folículos pilosos, cólon intestino, fígado, pulmão, linfócitos B/T, músculo (liso, cardíaco), ovário, pâncreas (células $\beta$ ), paratireoide, parótidas, pituitária, placenta, próstata, retina, pele, esperma, estômago, timo, tiroide, útero (Ferreira, 2013).

A função clássica da VD que é participar do controle da homeostase do cálcio e do fósforo. Quando a ingestão de cálcio é insuficiente, encontra-se uma dificuldade do organismo em manter as concentrações séricas do mineral adequadas. Nesta condição ocorre o estímulo para as glândulas paratireoides secretarem seu hormônio paratormônio (PTH), o qual tem o intuito de normalizar as concentrações séricas de cálcio, por meio do aumento da reabsorção óssea (liberação de cálcio dos ossos para o sangue), da redução da excreção urinária de cálcio e do aumento da atividade enzima 1-alfa-hidroxilase nos rins. A 1,25 (OH) VD formada em nível renal estimula absorção intestinal de cálcio por meio da interação com seu receptor nuclear VDR presentes nos enterósitos. Dessa maneira, com aumento da absorção estimulada pela VD associado ao consumo adequado de alimentos fontes de cálcio, sensores de cálcio presente nas glândulas paratireoide reconhece a normalização das concentrações séricas do mineral e, por conseguinte, a concentração de PTH também retorna aos valores normais por retrô alimentação negativa, feedback negativo, (Cozzolino \& Cominetti, 2013).

A vitamina D e o seu receptor também são importantes para o normal progresso do músculo esquelético e no aprimoramento da força e desempenho muscular. Em sua forma ativa, 1,25(OH)2D opera no músculo, através da ligação a dois VDRs (Ferreira, 2013). A vitamina D, especificamente no músculo, ativa a proteína cinase $\mathrm{C}$ e leva à libertação de cálcio, aumentando o pool de cálcio essencial para a contração muscular (Body et al., 2012).

Adams e Hewison (2010), articularam que a função evolutivamente mais primitiva da vitamina D é imunológica e criada para proteção do espaço interno do hospedeiro contra microorganismos invasores. Tal função é desempenhada pelo calcitriol sintetizado inicialmente por monócitos-macrófagos e age na via endócrina através da interação com VDRs, modulando a resposta imune inata. Quando produzido em quantidades suficientes, o calcitriol pode interagir e regular os perfis de citocinas de linfócitos T e B que expressam VDRs, no microambiente inflamatório local.

Em diante, a 1,25(OH)2D também atua nas células da mucosa intestinal gerando um aumento na absorção de cálcio, tanto na forma ativa no duodeno, quanto na passiva no jejuno. Na primeira, a interação da vitamina $\mathrm{D}$ e seu receptor instigam três mecanismos. Um deles é a expressão de proteínas responsáveis pela captação do cálcio, ocasionando o recrutamento dos canais deste pré-sintetizados na borda em escova dos enterócitos. Outro mecanismo permite a transferência desse íon através do enterócito, por meio de proteínas transportadoras de cálcio, como a calbidina. O terceiro induz os canais de membrana 
mediante uma bomba trifosfato de adenosina (ATP) dependente, facilitando a circulação para o fluido extracelular. No jejuno, proteínas capazes de formar canais de passagem intercelulares são ativadas, por onde ocorre a absorção passiva de acordo com a diferença do gradiente de concentração (Hewison, 2010; White, 2014).

O mecanismo renal pelo qual o calcitriol também age é parecido ao descrito no intestino. Na membrana a passagem do cálcio é admitida pelo aumento dos níveis do transportador de membrana e da proteína calbidina beneficiando o transporte transcelular (Thacher \& Clarke, 2011).

Outra função são os mecanismos subjacentes de ação da vitamina D na prevenção de doenças cardíacas que continuam explanados de maneira incompleta. Todavia, os mecanismos hipotetizados abrangem a presença de VDR em diversas células e sua possível modulação da expressão de inúmeros genes. A forma ativa $1,25(\mathrm{OH})_{2} \mathrm{D}_{3}$ interferem na cascata de reações e consequente na capacidade funcional de certas células. Tais mecanismos contêm a vitamina D como um regulador negativo para renina e um inibidor da proliferação celular e crescimento (Garcia \& Martini, 2010).

Russo (2017) cita que a vitamina D é caracterizada por ser fundamental para a homeostase, é responsável pela absorção de cálcio e de fósforo na região do intestino grosso, direcionada para os ossos e os rins, especialmente. Assegura o desempenho correto de músculos, nervos, desenvolvimento celular, emprego de energia, coagulação sanguínea, além também de ser importante na excreção de insulina, na resposta imunológica e outros aspectos e funções corporais.

E ainda que a carência da vitamina $\mathrm{D}$ em indivíduos normais pode estar colaborando para o desenvolvimento de doenças como: Fibromialgia, Doenças cardiovasculares, Hipertensão, Osteoporose, Intolerância à glicose: Diabetes Tipo I e II; Certas doenças infecciosas: Tuberculose, trato respiratório superior; Correlação inversa entre Síndrome Metabólica e níveis adequados de Vitamina D; Cânceres mais comuns: Intestino, mamas e próstata; Alterações no desenvolvimento e funções cerebrais; Doenças autoimunes: Esclerose múltipla, asma, artrite reumatoide, Doença de Crohn; Esquizofrenia: Carência de VD na gestação; Autismo; Déficit cognitivo; Nascimento de bebes com baixo peso; Hiperparatireoidismo secundário e primário; Depressão (Baixo nível sérico de VD e alto nível sérico de HPT); Doença de Alzheimer; Condições associadas à mortalidade infantil (Russo, 2017).

\subsection{Vitamina D e microbiota intestinal}

Nas últimas décadas, tanto o papel da vitamina D como a importância da integridade da microbiota intestinal para a saúde humana tem recebido destaque nas literaturas nacionais e mundial. Como pode ser observado pelo expressivo aumento do número de estudos em ambos os assuntos (Luthold, 2017). Diferente do que a maioria acredita a vitamina D não tem somente função na saúde óssea, sendo considerada um hormônio com papel fundamental no metabolismo e funções musculares, imuno e neurológicas (Clark \& Mach, 2016; Luthold, 2017).

A deficiência dessa vitamina tem sido associada à incidência de disbiose intestinal e aumento do risco de doenças crônicas e inflamatórias intestinais. O status adequado da vitamina D e a regulação dos receptores de vitamina D (VDR) desempenham um efeito modulador nas células imunes do intestino, podendo influenciar a composição da microbiota e a integridade da barreira intestinal (Clark \& Mach, 2016; Luthold, 2017).

A todo o momento, o trato gastrointestinal é exposto a compostos derivados de alimentos e microorganismos, sendo necessário um sistema de defesa complexo. Este é capaz de manter separado o conteúdo do lúmen intestinal dos demais tecidos, regular a absorção de nutrientes e permitir a interação entre a microbiota intestinal e o sistema imunológico. Evitando, dessa forma, que ocorra uma translocação de agentes infecciosos (Scaldaferri et al., 2012).

A barreira intestinal é composta por estruturas de adesão entre as células epiteliais adjacentes, entre elas, as junções apertadas e adesivas. Essas junções selam os espaços intercelulares e regulam a permeabilidade da barreira de mucosa. São estruturas altamente organizadas e formadas por complexos multiproteicos, que englobam proteínas como claudinas e 
occludina. As proteínas interagem com placas citosólicas da família Zonula Occludens (ZO) e cadeias, formando contatos celulares e células estáveis. Essa organização para estabilizar as células através da interação de proteínas (Cerulho, 2017).

Parte desse sistema de defesa se caracteriza por um epitélio intestinal diferenciado, que estabelece uma barreira com troca de moléculas entre o meio interno e o lúmen intestinal. Quando equilibrada, essa barreira é capaz de selecionar a passagem e proteger de partículas interpretadas pelo organismo como corpo estranho e de patógenos (Barbáchano et al., 2016).

Em pesquisas para analisar os fatores de influência e mecanismos envolvidos na permeabilidade da mucosa e da integridade da barreira intestinal é utilizado o indicador de resistência transepitelial da muscosa (TER). A identificação desses é importante para melhor entender e prevenir a interrupção da função de barreira que ocorre durante a inflamação e infecção bacteriana, facilitando a entrada de microorganismos no tecido. Dessa maneira, a função da barreira intestinal está vinculada à resposta imune, disbiose, inflamação e ao desenvolvimento de doenças inflamatórias intestinais (Barbáchano et al., 2016).

Embora o papel isolado das diferentes bactérias do trato gastrointestinal ainda não esteja bem definido, sabe-se que esse microbioma pode participar da fisiologia de doenças metabólicas, autoimunes e inflamatórias. Nesse sentido, compreender os determinantes e os fatores responsáveis por perturbações da sua composição, tanto na formação como na manutenção, é essencial para entender o estado de doença e desenvolver opções terapêuticas (Wang et al., 2016).

As investigações para determinar como a deficiência de vitamina D pode ser prejudicial ao sistema imune, interferindo na composição e papel da microbiota intestinal, favorecendo as doenças imunológicas e com inflamação subclínica, são recentes e ainda iniciais (Luthold, 2017).

A expressão do VDR é apontada como um regulador essencial da resposta imune, adaptada e adquirida, ao analisar a letalidade prematura de ratos com elevada concentração de Clostridium no intestino. Sugerindo que a expressão desse receptor é um fator crítico para a proteção contra infecção e manutenção da homeostase gastrointestinal (Chen et al., 2015).

\subsection{A relação entre doenças cardiovasculares, perfil intestinal e a deficiência de vitamina D}

Ao nível das doenças cardiovasculares (DCV) têm sido promovidos estudos que indicam possíveis mecanismos de ação ou influência da vitamina D ao nível do sistema cardiovascular (Santos, 2011 \& Lichtenstein et al., 2013). Segundo Barengolts (2013) e Adamczak et al. (2010), há também indícios da participação da vitamina D na homeostase do sistema cardiovascular por mediação da regulação microbiota e do estado inflamatório.

Entre as doenças cardiovasculares, a fibrilação atrial é um subtipo de arritmia cardíaca muito comum originada também pela deficiência da vitamina D. Estudos revisados demonstram que alto nível de vitamina D reduziu o risco de fibrilação arterial entre pessoas acima de 65 anos. A falta da vitamina está associada a um risco elevado de fibrilação arterial associado também a fibrilação atrial pós-operatória em pacientes submetidos à cirurgia de revascularização do miocárdio (Liu et al., 2019).

Ultimamente, a endotoxemia metabólica gerada pela microbiota intestinal tem sido associada a risco cardiometabólico aumentado. E devido à vitamina $\mathrm{D}$ exercer um papel expressivo na modulação do sistema imunológico no intestino, é possível que sua deficiência possa danificar a função da barreira intestinal favorecendo a translocação de endotoxinas, como lipopolissacarídeos (LPS) na circulação. LPS são conhecidos por promover inflamação sistêmica crônica, que predispõe à resistência à insulina. Inúmeros circulantes biomarcadores têm sido utilizados para avaliar a inflamação com objetivos clínicos (Luthold, 2017).

E ainda a presença da vitamina D em apropriadas concentrações inibe a proliferação de cardiomioblastos, promovendo a paragem do ciclo celular, aumenta a formação de cardiomicrotúbulos, sem indução do apoptose (Lichtenstein $e t$ al., 2013; Santos, 2011). 
Segundo Holick (2012) a vitamina D desempenha um efeito relaxante e consente maior flexibilidade aos vasos sanguíneos. Ela abranda os efeitos, sobre os vasos sanguíneos, do sistema renina-angiotensina, um sistema hormonal complexo que regula a pressão arterial e o equilíbrio da quantidade de água no corpo; além de trabalhar, diretamente, sobre os vasos e a musculatura lisa, relaxando-os. Por conseguinte, o sangue flui de modo mais eficaz, pois a pressão contra as paredes dos vasos é menor. Além disso, quando os níveis de 25 -vitamina D estão baixos, pode haver acúmulo de cálcio nas paredes arteriais, com a formação de placas de gordura perigosas. É a fragmentação dessas placas que ocasiona oclusões e trombos que são os responsáveis diretos dos infartos, dos acidentes vasculares cerebrais e da insuficiência cardíaca.

Do mesmo modo, os ossos podem ficar privados de cálcio, pois a deficiência de vitamina D impede a devida absorção do cálcio pelos intestinos. Esse fato implica risco duplo: ossos mais fracos e artérias enfermas, pois o cálcio se acumula na área errada. O endurecimento das artérias também é conhecido como aterosclerose (Holick, 2012).

Oliveira (2014) aborda que em relação à prevenção de DCV, já existia a hipótese há trinta anos que o aumento da incidência de DCV no inverno poderia ser uma consequência da deficiência ou insuficiência de vitamina D. E ao lado, foram encontrados os VDR no coração de rato, que instigaram a investigação de vitamina D e doenças cardiovasculares. Atualmente, um dos maiores estudos a respeito foi o terceiro National Health and Nutrition Examination Survey (NHANES III), um estudo transversal representativo da população não institucionalizada dos EUA feita entre 1988 e 1994, com mais de 12.000 pacientes, onde foi observada inversa relação entre: auto relato de angina, insuficiência cardíaca, infarto do miocárdio, pressão arterial com nível de vitamina D. E mais recente ainda, é o estudo onde os dados do NHANES III e NHANES 2001-2006 com mais de 270 mil participantes foram combinados, mostrando que os níveis mais baixos de vitamina D estão associados com o aumento da frequência cardíaca e da pressão arterial, concluindo que um baixo nível de vitamina D pode aumentar o trabalho cardíaco.

De acordo com Pavlović, Josipović e Pavlović (2011) o sistema renina-angiotensina-aldosterona (RAAS) é um regulador principal da pressão arterial e cumpre um papel crítico na regulação do volume e homeostase eletrolítica. O Sistema Renina Angiotensina (SRA) apresenta diversos componentes que interagem entre si, com a finalidade de manter o balanço hidroeletrolítico, de controlar a pressão sanguínea e arterial, assim como alguns processos metabólicos, crescimento e proliferação celular. A influência deste sistema nestes últimos processos foi descoberta recentemente. A ativação normal do SRA verifica-se em casos de Insuficiência cardíaca, em casos de restrição sódio e hipotensão (Santos et al., 2013).

Já o excesso de ativação pode acarretar problemas cardiovasculares, como hipertensão, enfartes ou arritmias, assim como a problemas renais ou metabólicos, como desenvolvimento de Diabetes Mellitus ou Síndrome metabólica (Pereira, 2014). E essa ativação parece ser o principal efeito decorrente ou associado à hipovitaminose D (Fonseca, 2015).

Em busca de verificar a ligação a vitamina D a hipertensão Borges (2020), em revisão integrativa expôs em seus resultados o mecanismo do SRA, onde em síntese da renina acontece nas células justaglomerulares dos rins e é responsável pela catálise do angiotênsinogênio em Angiotensina I, que depois será clivada em Angiotensina II, pele ECA (Enzima Conversora de Angiotensina). A angiotensina apresenta a capacidade de aumentar a pressão arterial de duas maneiras; atuando como um vasoconstritor e aumentando a resistência vascular periférica ou aumenteando a reabsorção renal de sódio pela produção de aldosterona (Rafaelli, 2015). A vitamina D opera impedindo a expressão gênica da renina, abrandando sua síntese e assim, impedindo a hiperestimulação desse sistema (Jorge et al., 2018; Carbone et al., 2014; Ajabshir, Asif \& Nayer, 2014).

A abastança de vitamina D também desempenha efeito protetor sobre a pressão arterial quando inibi o hormônio PTH, uma vez que concentrações elevadas desse hormônio estão relacionadas a diversos fatores de risco cardiovascular, dentre eles hipertensão (Fonseca, 2015).

E mesmo com uma alta prevalência de hipovitaminose D e estudos com pesquisas e estudos sobre a relação dela com DCV, ainda sim, a suplementação com vitamina D2 ou vitamina D3 é recomendada apenas para doentes com deficiência da mesma e populações com risco a essa deficiência (Maeda et al, 2014). O que segundo Holick et al. (2011), no presente 
momento, não há evidências suficientes para recomendar triagem para indivíduos que não estão em risco de deficiência, ou de prescrever vitamina D para alcançar níveis adequados de calcemia para a proteção cardiovascular.

A obtenção de vitamina D em humanos pode ser feita de diferentes fontes sendo elas: exposição solar, alimentação e suplementos. A exposição solar é considerada a forma mais fisiológica e primária no Brasil, devido ao clima tropical em boa parte de seu território e ao fato de que diferente de outros países no país não há políticas direcionadas para a fortificação de alimentos com vitamina D. E, são poucas as fontes naturais de vitamina D, com ressalto para o peixe (sardinha selvagem, salmão de cativeiro, sardinha em lata, cavala em lata, atum em lata), cogumelo shitake e gema de ovo. Outras fontes são o fígado de boi e de galinha, sucos cítricos, alimentos fortificas como a manteiga e leite integral (Andrade et al., 2015; Cozzolino \& Cominetti, 2013).

Cozzolino e Cominetti (2013), além disso, diz que é importante ressaltar que tanto a vitamina D2 quanto a D3 são encontradas nos alimentos e apresentam potencial de suprir as necessidades fisiológicas. E de modo geral, a vitamina D de origem alimentar é biodisponível durante as diferentes fases da vida. Andrade et al. (2015) por sua vez cita ainda que a ingestão de alimentos de origem marinha que fornecem a vitamina D deve ser incentivada. Dada a norma por pesquisas realizadas sob a influência da latitude e temperatura não aplicáveis à situação brasileira.

Dada essas relações, é importante lembrar que no Brasil, apesar de que a maior parcela da população more em regiões de apropriada exposição solar, a hipovitaminose D é um problema comum e não limitada somente mulheres menopausas e aos idosos, porém ainda acometendo adolescentes e crianças (Oliveira et al., 2013).

\section{Conclusão}

Após a análise é evidente que a vitamina D e microbiota exercem um papel essencial para homeostase do organismo, ambas têm uma forte relação que vem sendo evidenciada em estudos associados às doenças cardiovasculares. Assim como os mecanismos dos quais a hipovitaminose D e o desequilíbrio da microbiota se relacionam para aumento do risco de DCV.

Como a ligação entre a vitamina D e a barreira intestinal que tem sido associada ao enfraquecimento do sistema imunológico intestinal e favorecendo infecções e aumento de quadros de inflamação sistêmica que se relacionam como um fator risco para o desenvolvimento de doenças cardiométabolicas. Bem como a participação da vitamina D em sistemas diretamente ligados com o sistema cardiovascular como SRA, em que alguns estudos relatam a vitamina D em quantidade adequada como um fator de proteção para HAS.

E mesmo com a vasta variedade de autores relatando pontos de ligações entre causa e consequência a respeito do assunto, ainda são necessários muitos estudos e pesquisas que avaliem tal relação de maneira que esclareça os processos envolvendo a vitamina D, microbiota e sistema cardiovascular e seus respectivos papéis no risco cardiovascular aumentado, para que se possa estabelecer não apenas futuras linhas de tratamento, mas também meios de prevenção.

\section{Referências}

Adamczak, Daria Maria et al. (2014). The Role of Toll-Like Receptores and Vitamin D in Diabetes Mellitus Type 1- A Review. Scandinavian J Immunol, 80(2), 75-84.

Adams, J. S. \& Hewison, M. (2010). Update in vitamin D. J Clin Endocrinol Metab, 95(2), 471-478.

Ajabshir, S., Asif, A. \& Nayer, A. (2014). The effects of vitamin D on the renin-angiotensin system. J Nephropathol, 3(2), 41-3.

Ananthakrishnan, Ashwin N. et al. (2014). Higher plasma vitamin D is associated with reduced risk of Clostridium difficile infection in patients with inflammatory bowel diseases. Alimentary pharmacology and therapeutics, 39(10), 1136-1142.

Andrade, Paula Caroline de Oliveira et al. (2015). Alimentação, foto exposição e suplementação: influência nos níveis séricos de vitamina D. Rev Med Minas Gerais, 25(3), 432-437.

Barbáchano, Antonio et al. (2016). The endocrine vitamin D system in the gut. Molecular and Cellular Endocrinology. 
Barebgolts, Elena. (2013). Vitamin D And Prebiotics May Benefit The Intestinal Microbacteria And Improve Glucose Homeostasis In Prediabetes And Type 2 Diabetes. Endocr Pract, 19(3), 497-510.

Borges, Ana Clara do Nascimento et al. (2020). Vitamina D associada à hipertensão. Research, Society and Development, 9(1), 110911691.

Brasil. (2020). Ministério da Saúde. Secretaria de Vigilância em Saúde. Departamento de Análise em Saúde e Vigilância de Doenças Não Transmissíveis. Vigitel Brasil 2019: vigilância de fatores de risco e proteção para doenças crônicas por inquérito telefônico: estimativas sobre frequência e distribuição sociodemográfica de fatores de risco e proteção para doenças crônicas nas capitais dos 26 estados brasileiros e no Distrito Federal em 2019. 1 ed., Brasília, 137.

Brasil. (2020). Ministério da Saúde. Tabagismo custa R\$ 56,9 bilhões por ano ao Brasil. INCA, Instituto Nacional de Câncer-Ministério da Saúde, 2017. http://www2.inca.gov.br/wps/wcm/connect/agencianoticias/site/home/noticias/2017/tabagismo-custa-59-bilhoes-por-ano-ao-brasil.

Body, Jean Jacques et al. (2012). Extraskeletal benefits and risks of calcium, vitamin D and anti-osteoporosis medications. Osteoporos Int, $23(1), 1-23$.

Bourbon, Mafalda et al. (2016). Doenças cardiovasculares. Sabe como prevenir? Instituto Nacional de Saúde Doutor Ricardo Jorge. Departamento de Promoção da Saúde e Prevenção de Doenças Não Transmissíveis.

Carbone, Federico et al. (2014). Potential pathophysiological role for the vitamin D deficiency in essential hypertension. World J Cardiol, 6(5), 260-76.

Castro, Luiz Claudio Gonçalves de. (2011). O sistema endocrinológico vitamina D. Arq Bras Endocrinol Metab, 55(8), 566-575, http://www.scielo.br/scielo.php?script=sci_arttext\&pid=S0004-27302011000800010\&lng=pt\&nrm=iso.

Cerulho, Viviane Rodrigres. (2017). Vitamina D e saúde intestinal. 2017. Trabalho de Conclusão do Curso (Bacharelado em Nutrição) - Centro Universitário IBMR/Laurete International Universites, Rio de Janeiro.

Clark, A. \& Mach, N. Role of Vitamin D in the Hygiene Hypothesis: The Interplay. Frontiers in immunology, 7, 01-12.

Cozzolino, S. M. \& Cominetti, C. (2013). Bases bioquímicas e Fisiológicas da nutrição: nas diferentes fases da vida, na saúde e na doença. 1 ed., Baurueri: Manole, 1257.

Ferreira, Ana Carolina Freitas Reis. (2013). Vitamina D. Tese de mestrado (Mestrado Integrado em Medicina) - Faculdade de Medicina da Universidade de Coimbra, Coimbra.

Filho, A. J. I. \& Melamed, M. L. Vitamina D e doença renal. O que nós sabemos e o que nós não sabemos. J.Bras.Nefrol. , 35(4), 323-331, 2013. http://www.scielo.br/scielo.php?script=sci_arttext\&pid=S010128002013000400012\&lng=en\&nrm=iso.

Fonseca, Marília Izar Helfenstein. (2015). Hipertensão arterial, risco cardiovascular e vitamina D. Rev Bras Hipertens, 22(1), 2-8.

Garcia, V. C. \& Martini, L. A. (2010). Vitamin D and cardiovascular disease. Nutrients, 2(4), 426-37.

Hewison, M. (2010). Vitamin D and the imune system: new perspective on a old theme. Endocrinology Metabolism Clinics of North America, 39(2), 365-379.

Holick, Michael F. et al. (2011). Evaluation, treatment, and prevention of Vitamin D deficiency: An Endocrine Society Clinical Practice Guideline. Journal of Clinical Endocrinology \& Metabolism, 96(30), 1911.

Holick, Michael F. (2012). Vitamina D. 1 ed., São Paulo: Editora Fundamento Educacional Ltda, 320.

Jorge, Antonio José Lagoeiro et al. (2018). Vitamina D e doença cardiovascular. Int J Cardiovasc Sci. 31(4), 422-432.

Juonala, Markus et al. (2015). Childhood 25-OH vitamin D levels and carotid intima-media thickness in adulthood: the cardiovascular risk in Young Finns Study. J Clin Endocrinol Metab, 100(4), 1469-1476.

Kennel, K., Drake, M. \& Hurley, D. (2010). Vitamin D Deficiency in adults: when to test and how to treat. Mayo Clinic Proc, 85(8), 752-8.

Kich, Daiana Manuele et al. (2012). Determination of 25-hidroxy-vitamin D2 and D3 in plasma by HPLC-DAD. Jornal Brasileiro de Patologia e Medicina Laboratorial, v. 48, n. 5, p. 329-336. http://www.scielo.br/scielo.php?script=sci_arttext\&pid=S1676-24442012000500005\&lng=en\&nrm=iso.

Lichtenstein, Arnaldo et al. (2013). Vitamina D: ações extra ósseas e uso racional. Rev. Assoc. Med. Bras., 59(5), 495-506. http://www.scielo.br/scielo.php?script=sci_arttext\&pid=S0104-42302013000500015\&lng=en\&nrm=iso.

Liu, Xiao et al. (2019). The relationship between vitamin D and risk of atrial fibrillation: a dose-response analysis of observational studies. Nutrition journal, 18(1), 73 .

Ly, Nogoc P. el at. (2011). Gut microbiota, probiotics, and vitamin D: interrelated exposures influencing allergy, asthma, and obesity? J Allergy Clin Imтипоl, 127(5), 1087-1094.

Luthold, Renata Vidonscky. (2017). Vitamina D na modulação da microbiota intestinal: associações com os perfis inflamatório e cardiometabólico. Dissertação (Mestrado em Nutrição em Saúde Pública) - Faculdade de Saúde Pública, Universidade de São Paulo, São Paulo. Doi:10.11606/D.6.2017.tde06042017-095924.

Maeda, Sergio Setsuo et al. (2014). Recomendações da Sociedade Brasileira de Endocrinologia e Metabologia (SBEM) para o diagnóstico e tratamento da hipovitaminose D. Arq Bras Endocrinol Metabol, 58(5), 411-33.

Nadir, M. A., Szwejkowski, B. R. \& Witham, M. D. (2010). Vitamin D and Cardiovascular Prevention. Cardiovascular Therapeutics, 28(4), 5-12.

Norman, P. E. \& Powell J. T. (2014). Vitamin D and cardiovascular disease. Circ Res, 114(2), 379-393. 
Research, Society and Development, v. 10, n. 6, e29510616449, 2021

(CC BY 4.0) | ISSN 2525-3409 | DOI: http://dx.doi.org/10.33448/rsd-v10i6.16449

Oliveira, Renata M. S. et al. (2013). Association of vitamin D insuffi ciency with adiposity and metabolic disorders in Brazilian adolescents. Public Health Nutr, 17(4), 787-794.

Oliveira, Vanessa de et al. (2014). Influência da vitamina D na saúde humana. Acta Bioquímica Clínica Latinoamericana, 48(03), $339-347$.

Pavlović, D., Josipović, J. \& Pavlović, N. (2011). Vitamin D and hypertension. Period biol, 113, 3.

Pereira, Simão Pedro Torres. (2014). Sistema Renina Angiotensina, para além da Hipertensão Arterial. Dissertação (Mestrado em Ciências Farmacêuticas) Faculdade de Ciências da Saúde, Universidade Fernando Pessoa, Porto.

Rafaelli, Rafael Algusto et al. (2015). Influência da vitamina D nas doenças endocrinometabólicas. Semina: Ciências Biológicas e da saúde, 36(1), 333-348.

Rhodes, Jonathan Michael et al. (2020). Perspective: Vitamin D deficiency and COVID-19 severity - plausibly linked by latitude, ethnicity, impacts on cytokines, ACE2 and thrombosis. Journal of Internal Medicine, 289(1), 97-115.

Rother, Edna Terezinha. (2007). Revisão sistemática X revisão narrativa. Acta paul. Enferm, 20(2), 5-6.

Russo, Guilherme Vargas Gomes Almeida. (2017). A importância da vitamina D na sua saúde: uma revisão de literatura. International Journal of Science Dentistry, 2.

Santos, Robson A. S. et al. (2013). Angiotensin-converting enzyme 2, angiotensin-(1-7) and Mas: New players of the renin-angiotensin system. Journal of Endocrinology, 216, 1-17.

Santos, Alejandro. (2011). Papel da vitamina D no risco cardiovascular. Rev Facto de Ris, 20(9), 18-23.

Scaldaferri, Franco et al. (2012). The Gut Barrier: New Acquisitions and Therapeutic Approaches. Journal of Clinical Gastroenterology. $46(1), 12-17$.

Shoback, D., Sellmeyer, D. \& Bikle, D. (2011). Metabolic Bone Disease. In: GARDNER D. G.; SHOBACK, D. Greenspan's Basic and Clinical Endocrinology. 9th ed., New York: McGraw-Hill Companies. 227-284.

Thacher, T.D. \& Clarke, B.L. (2011). Vitamin D insufficiency. Mayo Clinic Proceedings, 86(01), 50-60.

Wang, Jun et al. (2016). Genome-wide association analysis identifies variation in vitamin D receptor and other host factors influencing the gut microbiota. Nature Genetics, 48(11), 1396-1406.

Weiss, Scott T. (2011). Bacterial componentes plus vitamin D: the ultimate solution to the asthma (autoimmune disease) edpidemic? J Allergy Clin Immunol, 127(5), 1128.

Weiss, S. T. \& Lintojua, A. A. (2015). Vitamin D, the gut microbiome, and the hygiene hipothesis. How does asthma begin? Am J Respir Crit Care Med, 191(5), 492-493.

White, Christopher. (2014). Update on vitamin D: More than just a nutrient. Obstetric Medicine, 7(01), 04-07.

Zittermann, A. \& Gummert, J. F. (2010). Nonclassical vitamin D action. Nutrients, 2(4), 408-25. 